\title{
Rapidity Factorization and TMDs
}

\author{
Andrey Tarasov* \\ Physics Department, Brookhaven National Laboratory, Bldg. 510A, Upton, NY 11973, U.S.A. \\ E-mail: atarasov@bnl.gov
}

To study transverse momentum distribution of particles produced in hadron-hadron collision one can use transverse momentum dependent (TMD) factorization approach. The TMD factorization is valid in the limit of infinitely small transverse momentum, i.e. when the transverse momentum of a produced particle is much smaller than its invariant mass. To apply the TMD factorization in the region of moderate transverse momentum one has to include power corrections. We will discuss how one can calculate power corrections to TMD factorization through the solution of the equations of motion in background fields of the colliding hadrons.

QCD Evolution 2017

22-26 May, 2017

Jefferson Lab Newport News, VA - USA

${ }^{*}$ Speaker. 


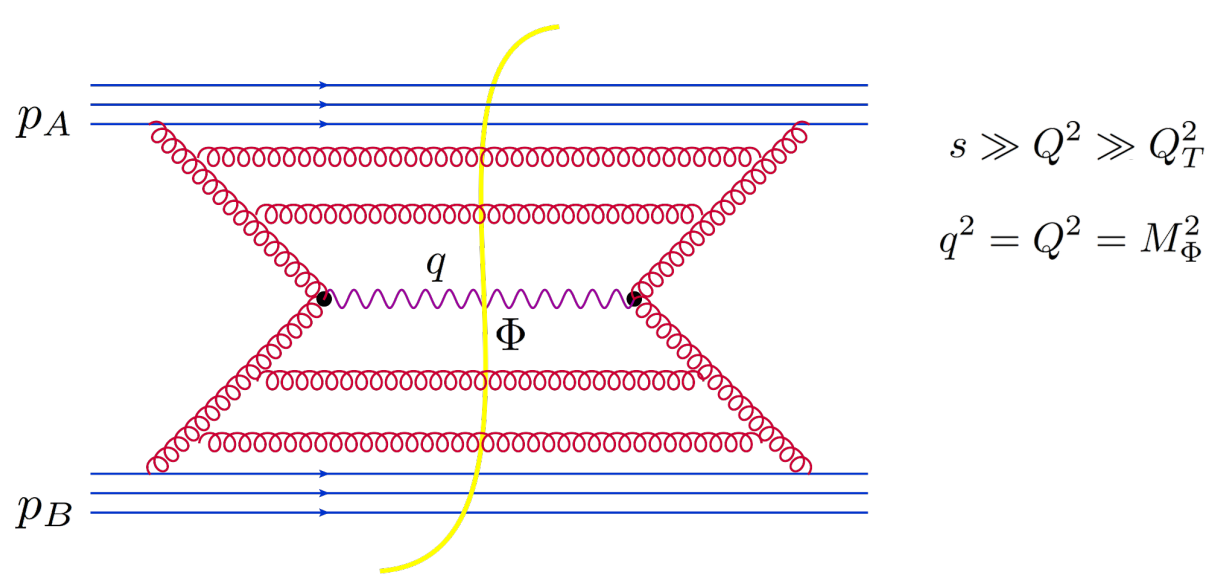

Figure 1: Particle production by gluon-gluon fusion

\section{Introduction}

To discuss a general scheme of calculation of higher-twist corrections to TMD factorization, let us consider a relatively simple case of scalar particle $\Phi$ production (e.g. Higgs particle) in hadron-hadron collision through the gluon-gluon fusion, which is defined by interaction vertex

$$
\mathscr{L}_{\Phi}=g_{\Phi} \int d^{4} x \Phi(x) g^{2} F_{\mu v}^{a}(x) F^{a \mu v}(x)
$$

where $F_{\mu v}(x)$ is the gluon field strength tensor.

A typical diagram for particle $\Phi$ production with momentum $q$ is presented in Fig.1. It is straightforward to calculate the corresponding differential cross section:

$$
d \sigma=\frac{d^{3} q}{2 E_{q}(2 \pi)^{3}} \frac{g_{\Phi}^{2}}{2 s} W\left(p_{A}, p_{B}, q\right)
$$

where $p_{A}$ and $p_{B}$ are momenta of incoming hadrons, $s=\left(p_{A}+p_{B}\right)^{2}$ and the hadronic tensor $W\left(p_{A}, p_{B}, q\right)$ is defined as

$$
W\left(p_{A}, p_{B}, q\right) \stackrel{\text { def }}{=} \int d^{4} x e^{-i q x}\left\langle p_{A}, p_{B}\left|g^{4} F^{2}(x) F^{2}(0)\right| p_{A}, p_{B}\right\rangle
$$

where we use a shorthand notation $F^{2}(x) \equiv F_{\mu \nu}^{a}(x) F^{a \mu \nu}(x)$.

The goal of this paper is to calculate the hadronic tensor (1.3) in the leading order (tree level diagrams) and show that it is a convolution of two TMD operators describing non-perturbative distribution of gluons in colliding hadrons [1, 2, 3, 4].

\section{Rapidity factorization approach}

Factorization principle implies that one can separate different types of emission contributing to an experimental observable, in our case the hadronic tensor (1.3), and consider them independently. 


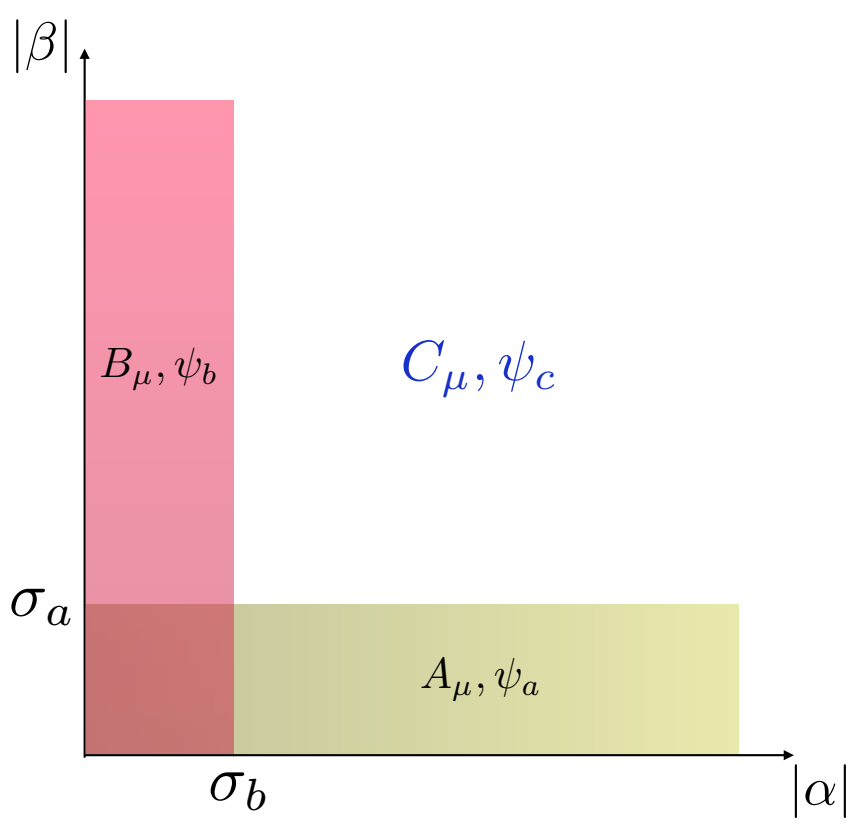

Figure 2: Three types of the fields

As a result, before derivation of the factorization theorem one should define those types of emission in a rigorous way. In this paper we will use the rapidity factorization scheme [5] which separates the fields based on the values of the Sudakov variables $\alpha$ and $\beta$, which define the standard decomposition of an arbitrary momentum vector $p=\alpha p_{1}+\beta p_{2}+p_{\perp}$, where $p_{1}^{\mu}=p_{A}^{\mu}-\left(p_{A}^{2} / s\right) p_{B}^{\mu}$ and $p_{2}^{\mu}=p_{B}^{\mu}-\left(p_{B}^{2} / s\right) p_{A}^{\mu}$ are light-like vectors. We will also use notations $x_{\bullet} \equiv x_{\mu} p_{1}^{\mu}$ and $x_{*} \equiv x_{\mu} p_{2}^{\mu}$ for the dimensionless light-cone coordinates.

To separate different types of emission we introduce two cut-off parameters, $\sigma_{a}$ and $\sigma_{b}$, and define three types of fields. The fields $A_{\mu}, \psi_{a}$ with $\beta<\sigma_{a}$ describe emission which is collinear to momentum vector $p_{A}$ and can be associated with dynamics of the "projectile" hadron $A$. In a similar manner, the fields $B_{\mu}, \psi_{B}$ with $\alpha<\sigma_{b}$ correspond to emission collinear to momentum $p_{B}$ and describe the structure of the "target" hadron $B$. Finally the "cental" region of emision is constructed from fields $C_{\mu}, \psi_{C}$ with $\alpha>\sigma_{b}$ and $\beta>\sigma_{a}$. It is obvious that fields of $C$ sector can be created through interaction of $A$ and $B$ fields only. A schematic representation of separation of fields into three sectors is presented in Fig. 2.

In terms of fields $A, B$ and $C$ the process of particle production in hadron-hadron collision can be quite involved, see Fig. 3. To prove factorization, one should show that complicated interaction between sectors can be disentangled and presented as a convolution of independent pieces each constructed from fields of only one sector.

To separate contributions of different sectors to hadronic tensor (1.3) we will use its functional integral representation:

$$
W\left(p_{A}, p_{B}, q\right)=\sum_{X} \int d^{4} x e^{-i q x}\left\langle p_{A}, p_{B}\left|g^{2} F^{2}(x)\right| X\right\rangle\left\langle X\left|g^{2} F^{2}(0)\right| p_{A}, p_{B}\right\rangle
$$




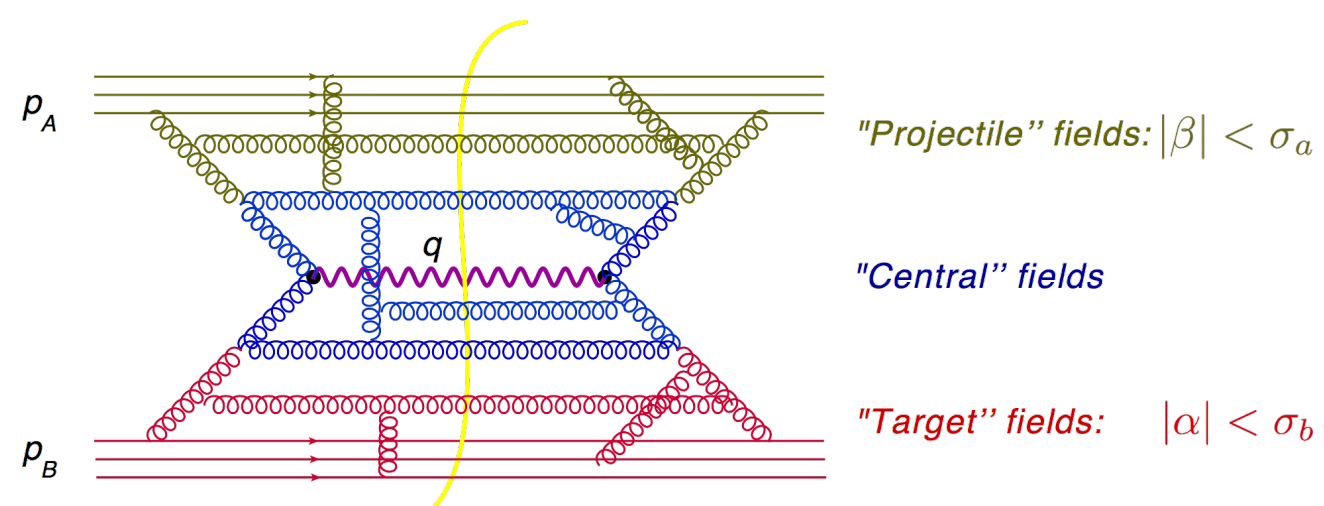

Figure 3: Particle production by gluon-gluon fusion

$$
\begin{aligned}
& =\lim _{t_{i} \rightarrow-\infty}^{t_{f} \rightarrow \infty} g^{4} \int d^{4} x e^{-i q x} \int^{\tilde{A}\left(t_{f}\right)=A\left(t_{f}\right)} D \tilde{A} \mu A_{\mu} \int^{\tilde{\psi}\left(t_{f}\right)=\psi\left(t_{f}\right)} D \tilde{\tilde{\psi}} D \tilde{\psi} D \bar{\psi} D \psi \Psi_{p_{A}}^{*}\left(\overrightarrow{\tilde{A}}\left(t_{i}\right), \tilde{\psi}\left(t_{i}\right)\right) \\
& \times \Psi_{p_{B}}^{*}\left(\overrightarrow{\tilde{A}}\left(t_{i}\right), \tilde{\psi}\left(t_{i}\right)\right) e^{-i S_{\mathrm{QCD}}(\tilde{A}, \tilde{\psi})} e^{i S_{\mathrm{QCD}}(A, \psi)} \tilde{F}^{2}(x) F^{2}(0) \Psi_{p_{A}}\left(\vec{A}\left(t_{i}\right), \psi\left(t_{i}\right)\right) \Psi_{p_{B}}\left(\vec{A}\left(t_{i}\right), \psi\left(t_{i}\right)\right)
\end{aligned}
$$

where functional integral over fields $A, \psi$ represent amplitude $\left\langle X\left|F^{2}(0)\right| p_{A}, p_{B}\right\rangle$, while integration over fields $\tilde{A}, \tilde{\psi}$ corresponds to complex conjugate amplitude $\left\langle p_{A}, p_{B}\left|F^{2}(x)\right| X\right\rangle$. The hadrons' wave functions at initial time $t_{i}$ are $\Psi_{p_{A}}\left(\vec{A}\left(t_{i}\right), \psi\left(t_{i}\right)\right)$ and $\Psi_{p_{B}}\left(\vec{A}\left(t_{i}\right), \psi\left(t_{i}\right)\right)$. The boundary conditions for the functional integral $\tilde{A}\left(t_{f}\right)=A\left(t_{f}\right)$ and $\tilde{\psi}\left(t_{f}\right)=\psi\left(t_{f}\right)$ represent sum over complete set of final states $\sum_{X}$, cf. Refs. [10, 11].

Indeed, in terms of the functional integral separation of fields into three sectors is straightforward:

$$
\begin{aligned}
& W\left(p_{A}, p_{B}, q\right)=g^{4} \int d^{4} x e^{-i q x} \int^{\tilde{A}\left(t_{f}\right)=A\left(t_{f}\right)} D \tilde{A}_{\mu} D A_{\mu} \int^{\tilde{\psi}_{a}\left(t_{f}\right)=\psi_{a}\left(t_{f}\right)} D \bar{\psi}_{a} D \psi_{a} D \tilde{\bar{\psi}}_{a} D \tilde{\psi}_{a} \\
& \times e^{-i S_{\mathrm{QCD}}\left(\tilde{A}, \tilde{\psi}_{a}\right)} e^{i S_{\mathrm{QCD}}\left(A, \psi_{a}\right)} \Psi_{p_{A}}^{*}\left(\overrightarrow{\tilde{A}}\left(t_{i}\right), \tilde{\psi}_{a}\left(t_{i}\right)\right) \Psi_{p_{A}}\left(\vec{A}\left(t_{i}\right), \psi\left(t_{i}\right)\right) \\
& \times \int^{\tilde{B}\left(t_{f}\right)=B\left(t_{f}\right)} D \tilde{B}_{\mu} D B_{\mu} \int^{\tilde{\psi}_{b}\left(t_{f}\right)=\psi_{b}\left(t_{f}\right)} D \bar{\psi}_{b} D \psi_{b} D \tilde{\bar{\psi}}_{b} D \tilde{\psi}_{b} \\
& \times e^{-i S_{\mathrm{QCD}}\left(\tilde{B}, \tilde{\psi}_{b}\right)} e^{i S_{\mathrm{QCD}}\left(B, \psi_{b}\right)} \Psi_{p_{B}}^{*}\left(\overrightarrow{\tilde{B}}\left(t_{i}\right), \tilde{\psi}_{b}\left(t_{i}\right)\right) \Psi_{p_{B}}\left(\vec{B}\left(t_{i}\right), \psi_{b}\left(t_{i}\right)\right) \\
& \times \int D C_{\mu} \int^{\tilde{C}\left(t_{f}\right)=C\left(t_{f}\right)} D \tilde{C}_{\mu} \int D \bar{\psi}_{C} D \psi_{C} \int^{\tilde{\psi_{c}}\left(t_{f}\right)=\psi_{c}\left(t_{f}\right)} D \tilde{\bar{\psi}}_{C} D \tilde{\psi}_{C} \tilde{F}_{C}^{2}(x) F_{C}^{2}(0) e^{-i \tilde{S}_{C}+i S_{C}}
\end{aligned}
$$

where action $S_{C}=S_{\mathrm{QCD}}(A+B+C)-S_{\mathrm{QCD}}(A)-S_{\mathrm{QCD}}(B)$ describes dynamics of fields $C_{\mu}, \psi_{C}$ in the background of external fields $A_{\mu}, \psi_{a}$ and $B_{\mu}, \psi_{b}$.

From the form of this action it is obvious that in general functional integrals over $A$ and $B$ fields depend on each other through interaction with $C$ fields. To prove factorization we need to show that integral over $C$ fields can be reduced to a product of two operators each constructed from $A$ or $B$ fields only. As a result the hadronic tensor $W$ can be written as a product of independent functional integrals over $A$ and $B$ fields representing target and projectile TMD operator.

The functional integral over fields of the "central" sector has a general form

$$
\int D C_{\mu} \int^{\tilde{C}\left(t_{f}\right)=C\left(t_{f}\right)} D \tilde{C}_{\mu} \int D \bar{\psi}_{C} D \psi_{C} \int^{\tilde{\psi}_{c}\left(t_{f}\right)=\psi_{c}\left(t_{f}\right)} D \tilde{\Psi}_{C} D \tilde{\psi}_{C} g^{4} \tilde{F}_{C}^{2}(x) F_{C}^{2}(0) e^{-i \tilde{S}_{C}+i S_{C}}
$$




$$
=e^{S_{\mathrm{eff}}(A, B, \tilde{A} \tilde{B})} \mathscr{O}\left(q, x ; A, \tilde{A}, \psi_{a} \tilde{\psi}_{a} ; B, \tilde{B}, \psi_{b}, \tilde{\psi}_{b}\right)
$$

where operator $\mathscr{O}$ is constructed from diagrams of fields $C$ connected to $\tilde{F}_{C}^{2}(x) F_{C}^{2}(0)$ in the background of $A$ and $B$ and action $S_{\text {eff }}$ represents exponentiation of direct interaction between spectators. This interaction is mediated by quark-gluon exchanges with $q^{2} \simeq-q_{\perp}^{2}$ and provides Glauber contribution to hadronic tensor $W\left(p_{A}, p_{B}, q\right)$.

The resummation of Glauber exchanges is an open problem. The form of the effective action $S_{e f f}$ is known only in first few orders orders of perturbation theory, see [5, 6, 7, 8, 9]. The remarkable property of the known result is that

$$
\left.e^{S_{\text {eff }}(A, B, \tilde{A}, \tilde{B})}\right|_{A=\tilde{A} ; B=\tilde{B}}=1
$$

In our problem, the fields at different sides of the cut are equivalent to each other, i.e. $A=\tilde{A}$ and $B=\tilde{B}$, due to boundary conditions in the functional integral (2.2) and the form of background fields dependence on coordinates.

Indeed, in the tree level approximation we don't have large logarithms of the cut-off parameters $\sigma_{a}$ and $\sigma_{b}$. As a result one can assume $\sigma_{a}, \sigma_{b}=0$. In other words, in any integral over momentum the contour of integration can be deformed away from the region of small $\alpha$ and $\beta$ where the values of the cut-off parameters are important.

The choice of $\sigma_{a}, \sigma_{b}=0$ means that the background fields $A$ don't cary any $\beta$ component of the transverse momenta. On the other hand fields of the $B$ sector don't bring any fraction $\alpha$ of the momentum $p_{1}$. This corresponds to the following choice of the dependence of the background fields on coordinates:

$$
\begin{array}{ll}
A(x)=A\left(x_{\bullet}, x_{\perp}\right), & \psi_{a}(x)=\psi_{a}\left(x_{\bullet}, x_{\perp}\right) \\
B(x)=B\left(x_{*}, x_{\perp}\right), & \psi_{b}(x)=\psi_{b}\left(x_{*}, x_{\perp}\right)
\end{array}
$$

Now let us take into account the boundary conditions in the functional integral (2.2):

$$
\tilde{A}\left(t_{f}\right)=A\left(t_{f}\right), \quad \tilde{\psi}_{a}\left(t_{f}\right)=\psi_{a}\left(t_{f}\right) \text { and } \quad \tilde{B}\left(t_{f}\right)=B\left(t_{f}\right), \quad \tilde{\psi}_{b}\left(t_{f}\right)=\psi_{b}\left(t_{f}\right)
$$

The fields $A, \psi_{a}$ and $\tilde{A}, \tilde{\psi}_{a}$ do not depend on $x_{*}$, so if they coincide at $x_{*} \rightarrow \infty$ they have to be the same. The same logic can be applied to $B, \psi_{b}$ and $\tilde{B}, \tilde{\psi}_{b}$ fields which do not depend on $x_{\bullet}$. As a result we have

$$
\begin{array}{ll}
A\left(x_{\bullet}, x_{\perp}\right)=\tilde{A}\left(x_{\bullet}, x_{\perp}\right), & \psi_{a}\left(x_{\bullet}, x_{\perp}\right)=\tilde{\psi}_{a}\left(x_{\bullet}, x_{\perp}\right) \\
B\left(x_{*}, x_{\perp}\right)=\tilde{B}\left(x_{*}, x_{\perp}\right), & \psi_{b}\left(x_{*}, x_{\perp}\right)=\tilde{\psi}_{b}\left(x_{*}, x_{\perp}\right)
\end{array}
$$

which leads to cancellation of the Glauber contribution, see eq. (2.4).

Now we see that with our choice of the background fields we need to calculate diagrams connected to $\tilde{F}_{C}^{2}(x) F_{C}^{2}(0)$, i.e.

$$
\begin{aligned}
& \int D C_{\mu} \int^{\tilde{C}\left(t_{f}\right)=C\left(t_{f}\right)} D \tilde{C}_{\mu} \int D \bar{\psi}_{C} D \psi_{C} \int^{\tilde{\psi}_{c}\left(t_{f}\right)=\psi_{c}\left(t_{f}\right)} D \tilde{\psi}_{C} D \tilde{\psi}_{C} g^{4} \tilde{F}_{C}^{2}(x) F_{C}^{2}(0) e^{-i \tilde{S}_{C}+i S_{C}} \\
& =\mathscr{O}\left(q, x ; A, \psi_{a} ; B, \psi_{b}\right)
\end{aligned}
$$

In next sections we will see that calculation of the functional integral (2.8) is equivalent to solution of the Yang-Mills equation of motion with retarded Green functions. We will see how one can construct this solution using parametrization of the background fields. 


\section{Classical equations of motion}

In this paper we discuss calculation of the functional integral $\mathscr{O}$ at the tree level and do not consider diagrams with loops which generate evolution of projectile and target TMD operators.

It is well known that resummation of Feynman diagrams at the tree level is equivalent to solution of equations of motion

$$
\begin{aligned}
& D^{v} F_{\mu \nu}^{a}(\bar{A}+\bar{B}+C)=g \sum_{f}\left(\bar{\psi}_{a}^{f}+\bar{\psi}_{b}^{f}+\bar{\psi}_{c}^{f}\right) \gamma_{\mu} t^{a}\left(\psi_{a}^{f}+\psi_{b}^{f}+\psi_{c}^{f}\right) \\
& (i \not \partial+g \bar{A}+g \bar{B}+g \not C)\left(\psi_{a}^{f}+\psi_{b}^{f}+\psi_{c}^{f}\right)=m\left(\psi_{a}^{f}+\psi_{b}^{f}+\psi_{c}^{f}\right)
\end{aligned}
$$

In general, the perturbative solution of eq. (3.1) should be constructed with Feynman propagators. However, calculation of the double functional integral (2.8) is equivalent to calculation of amplitudes with retarded Green functions. This can be shown explicitly by calculation of Feynman diagrams with the cut at the tree level and resummation of diagrams with different position of the cut.

Solution of equations of motion (3.1) for fields $C, \psi_{c}$ in background fields $A, \psi_{a}$ and $B, \psi_{b}$ with retarded Green functions leads to solution with vanishing fields, i.e. $C_{\mu} \rightarrow 0$ and $\psi_{c} \rightarrow \infty$, at $t \rightarrow-\infty$, which is equivalent to solution of (3.1) with boundary conditions

$$
\begin{array}{ll}
A_{\mu}(x) \stackrel{x_{*} \rightarrow-\infty}{=} \bar{A}_{\mu}\left(x_{\bullet}, x_{\perp}\right), & \psi(x) \stackrel{x_{*} \rightarrow-\infty}{=} \psi_{a}\left(x_{\bullet}, x_{\perp}\right) \\
A_{\mu}(x) \stackrel{x_{\bullet}}{\stackrel{\rightarrow}{=}}=\bar{B}_{\mu}\left(x_{*}, x_{\perp}\right), & \psi(x) \stackrel{x_{\bullet}}{=} \psi_{b}\left(x_{*}, x_{\perp}\right)
\end{array}
$$

where we use a shorthand notation $A=\bar{A}+\bar{B}+C$ and $\psi=\psi_{a}+\psi_{b}+\psi_{c}$. The physical meaning of this boundary conditions is absence of "centra" fields $C_{\mu}, \psi_{c}$ before collision of hadrons.

Unfortunately, we can not solve equations of motion (3.1) in general case. However, in case of large center of mass energy of the scattering reaction we can construct an approximate solution.

Indeed, at $s \rightarrow \infty$ components of the background fields are not equivalent to each other. Let us introduce the following parametrization for gluon background fields

$$
\begin{aligned}
& \bar{A}_{*}\left(x_{\bullet}, x_{\perp}\right) \sim s, \quad \bar{A}_{\bullet}\left(x_{\bullet}, x_{\perp}\right) \sim m_{\perp}^{2}, \quad \bar{A}_{i}\left(x_{\bullet}, x_{\perp}\right) \sim m_{\perp} \\
& \bar{B}_{*}\left(x_{*}, x_{\perp}\right) \sim m_{\perp}^{2}, \quad \bar{B}_{\bullet}\left(x_{*}, x_{\perp}\right) \sim s, \quad \bar{B}_{i}\left(x_{*}, x_{\perp}\right) \sim m_{\perp}
\end{aligned}
$$

where $m_{\perp}$ is a typical small hadronic mass, and analogous parametrization for quark fields as well

$$
\begin{aligned}
& p_{1} \psi_{a}\left(x_{\bullet}, x_{\perp}\right) \sim m_{\perp}^{5 / 2}, \quad \gamma_{i} \psi_{a}\left(x_{\bullet}, x_{\perp}\right) \sim m_{\perp}^{3 / 2}, \quad p_{2} \psi_{a}\left(x_{\bullet}, x_{\perp}\right) \sim s \sqrt{m_{\perp}} \\
& p_{1} \psi_{b}\left(x_{*}, x_{\perp}\right) \sim s \sqrt{m_{\perp}}, \quad \gamma_{i} \psi_{b}\left(x_{*}, x_{\perp}\right) \sim m_{\perp}^{3 / 2}, \quad p_{2} \psi_{b}\left(x_{*}, x_{\perp}\right) \sim m_{\perp}^{5 / 2}
\end{aligned}
$$

It is obvious that this parametrization introduces a small parameter $m_{\perp}^{2} / s$ which we will use to construct an approximate solution of the equations of motion at $s \rightarrow \infty$.

Indeed, let us look at perturbative solution of the equations of motion (3.1):

$$
\begin{aligned}
A_{\mu}^{[1] a}(x)= & \int d^{4} z\left(x\left|\frac{1}{\mathscr{P}^{2} g^{\mu v}+2 i g \mathscr{F}[0] \mu v}\right| z\right)^{a b} L^{b v}(z) \\
A_{\mu}^{[2] a}(x)= & g \int d^{4} z\left[-i\left(x\left|\frac{1}{\mathscr{P}^{2} g^{\mu \eta}+2 i g \mathscr{F}[0] \mu \eta} \mathscr{P}^{\xi}\right| z\right)^{a a^{\prime}} f^{a^{\prime} b c} A_{\xi}^{[1] b} A^{[1] c \eta}\right. \\
& \left.+\left(x\left|\frac{1}{\mathscr{P}^{2} g^{\mu \eta}+2 i g \mathscr{F}[0] \mu \eta}\right| z\right)^{a a^{\prime}} f^{a^{\prime} b c} A^{[1] b \xi}\left(\mathscr{D} \xi A^{[1] c \eta}-\mathscr{D}^{\eta \eta} A_{\xi}^{[1] c}\right)\right]
\end{aligned}
$$




$$
\Psi_{f}^{[1]}(x)=-\int d^{4} z\left(x\left|\frac{1}{\not \supset}\right| z\right) L_{\psi}(z), \quad \Psi_{f}^{[2]}(x)=-g \int d^{4} z\left(x\left|\frac{1}{\not \supset}\right| z\right) \not A^{[1]}(z) \Psi_{f}^{[0]}(z)
$$

where zero-order approximation is a sum of background fields

$$
\begin{aligned}
& g \mathscr{A}_{\mu}^{[0]}(x)=\bar{A}_{\mu}\left(x_{\bullet}, x_{\perp}\right)+\bar{B}_{\mu}\left(x_{*}, x_{\perp}\right) \\
& \Psi^{[0]}(x)=\psi_{a}\left(x_{\bullet}, x_{\perp}\right)+\psi_{b}\left(x_{*}, x_{\perp}\right)
\end{aligned}
$$

and the linear term

$$
\begin{aligned}
& L_{i}^{a} \equiv \mathscr{D}^{\mu} \mathscr{F}_{\mu i}^{[0] a}+g \bar{\Psi}^{[0]} \gamma_{i} t^{a} \Psi^{[0]} \\
& L_{\psi} \equiv \not P \Psi^{[0]}
\end{aligned}
$$

defines creation of "central" sector fields $C, \psi_{c}$ through interaction of background fields $\mathscr{A}_{\mu}^{[0]}, \Psi^{[0]}$.

The perturbative solutions (3.5) and (3.6) contains retarded Green functions which one can understand as expansion

$$
\begin{aligned}
& \left(x\left|\frac{1}{\bar{P}^{2} g^{\mu v}+2 i g \bar{F}^{\mu v}+i \varepsilon p_{0}}\right| y\right) \equiv\left(x\left|\frac{1}{p^{2}+i \varepsilon p_{0}}\right| y\right)-g\left(x\left|\frac{1}{p^{2}+i \varepsilon p_{0}} \mathscr{O}_{\mu v} \frac{1}{p^{2}+i \varepsilon p_{0}}\right| y\right) \\
& +g^{2}\left(x\left|\frac{1}{p^{2}+i \varepsilon p_{0}} \mathscr{O}_{\mu \xi} \frac{1}{p^{2}+i \varepsilon p_{0}} \mathscr{O}_{v}^{\xi} \frac{1}{p^{2}+i \varepsilon p_{0}}\right| y\right)+\ldots
\end{aligned}
$$

where

$$
\begin{aligned}
& \bar{P}_{\mu} \equiv i \partial_{\mu}+g \bar{A}_{\mu}+g \bar{B}_{\mu}, \quad \bar{F}_{\mu \nu}=\partial_{\mu}(\bar{A}+\bar{B})_{\nu}-\mu \leftrightarrow v-i g\left[\bar{A}_{\mu}+\bar{B}_{\mu}, \bar{A}_{v}+\bar{B}_{v}\right] \\
& \mathscr{O}_{\mu \nu} \equiv\left(\left\{p^{\xi}, \bar{A}_{\xi}+\bar{B}_{\xi}\right\}+g(\bar{A}+\bar{B})^{2}\right) g_{\mu \nu}+2 i \bar{F}_{\mu \nu}
\end{aligned}
$$

It is easy to see that in perturbative solutions (3.5), (3.6) any operator is constructed from background fields and has certain power counting $\left(m_{\perp}^{2} / s\right)^{n}$, which can be used to separate leading contribution.

In the next section we will see results of this separation with the specific choice of the gauge of the background fields. Yet the final result of calculation of the hadronic tensor $W\left(p_{A}, p_{B}, q\right)$ will be gauge invariant.

\section{Parametrization of the perturbative solution}

Before we perform parametrization of the perturbative solution and separation of the leading contribution let us rotate background field $A, \psi_{a}$ and $B, \psi_{b}$ to a new set of fields $U, \Sigma_{a}$ and $V, \Sigma_{b}$ correspondingly using gauge matrix $\Omega$.

It is convenient to work in a gauge with

$$
U_{*}=0, \quad V_{\bullet}=0
$$

where we don't have large components of the fields. 
The existence of such gauge rotation has been explicitly shown in [12]. In this case the gauge matrix has a remarkable property

$$
\Omega\left(x_{*}, x_{\bullet}, x_{\perp}\right) \stackrel{x_{*} \rightarrow-\infty}{\rightarrow}\left[x_{\bullet},-\infty\right]_{\bullet}^{\bar{A}_{*}}, \quad \Omega\left(x_{*}, x_{\bullet}, x_{\perp}\right) \stackrel{x_{\bullet} \rightarrow-\infty}{\rightarrow}\left[x_{*},-\infty_{*}\right]_{x}^{\bar{B}_{\bullet}}
$$

The rotated background fields satisfy equations of motion

$$
\begin{aligned}
& D_{U}^{v} U_{\mu \nu}^{a}=g^{2} \sum_{f} \bar{\Sigma}_{a}^{f} \gamma_{\mu} t^{a} \Sigma_{a}^{f}, \quad i \not D_{U} \Sigma_{a}=0 \\
& D_{V}^{v} V_{\mu \nu}^{a}=g^{2} \sum_{f} \bar{\Sigma}_{b}^{f} \gamma_{\mu} t^{a} \Sigma_{b}^{f}, \quad i \not D_{V} \Sigma_{b}=0
\end{aligned}
$$

and have the following parametrization

$$
U_{\bullet} \sim V_{*} \sim m_{\perp}^{2}, \quad U_{i} \sim V_{i} \sim m_{\perp}
$$

We see that rotated fields don't have large components proportional to $s$.

In terms of new background fields we need to solve equations of motion (3.1) with boundary conditions

$$
\begin{aligned}
& g A_{\mu}(x) \stackrel{x_{*}}{\stackrel{\rightarrow}{=}}=U_{\mu}\left(x_{\bullet}, x_{\perp}\right), \quad \psi(x) \stackrel{x_{*}}{\stackrel{\rightarrow}{=}} \Sigma_{a}\left(x_{\bullet}, x_{\perp}\right) \\
& g A_{\mu}(x) \stackrel{x_{\bullet}}{\stackrel{\rightarrow}{=}}=V_{\mu}\left(x_{*}, x_{\perp}\right), \quad \psi(x) \stackrel{x_{\bullet} \rightarrow-\infty}{=} \Sigma_{b}\left(x_{*}, x_{\perp}\right)
\end{aligned}
$$

where

$$
\begin{aligned}
U_{\mu}\left(x_{\bullet}, x_{\perp}\right) & \equiv \frac{2}{s} p_{2 \mu} U_{\bullet}\left(x_{\bullet}, x_{\perp}\right)+U_{\mu_{\perp}}\left(x_{\bullet}, x_{\perp}\right) \\
V_{\mu}\left(x_{*}, x_{\perp}\right) & \equiv \frac{2}{s} p_{1 \mu} V_{*}\left(x_{*}, x_{\perp}\right)+V_{\mu_{\perp}}\left(x_{*}, x_{\perp}\right) \\
U_{i}\left(x_{\bullet}, x_{\perp}\right) & \equiv \frac{2}{s} \int_{-\infty}^{x_{\bullet}} d x_{\bullet}^{\prime} U_{* i}\left(x_{\bullet}^{\prime}, x_{\perp}\right), \quad V_{i}\left(x_{*}, x_{\perp}\right) \equiv \frac{2}{s} \int_{-\infty}^{x_{*}} d x_{*}^{\prime} V_{\bullet i}\left(x_{*}^{\prime}, x_{\perp}\right) \\
U_{\bullet}\left(x_{\bullet}, x_{\perp}\right) & \equiv \frac{2}{s} \int_{-\infty}^{x_{\bullet}} d x_{\bullet}^{\prime} U_{* \bullet}\left(x_{\bullet}^{\prime}, x_{\perp}\right), \quad V_{*}\left(x_{*}, x_{\perp}\right) \equiv-\frac{2}{s} \int_{-\infty}^{x_{*}} d x_{*}^{\prime} V_{* \bullet}\left(x_{*}^{\prime}, x_{\perp}\right)
\end{aligned}
$$

and $\Sigma_{a}, \Sigma_{b}$ are defined as

$$
\Sigma_{a}\left(z_{\bullet}, z_{\perp}\right) \equiv\left[-\infty_{\bullet}, z_{\bullet}\right]_{z} \psi_{a}\left(z_{\bullet}, z_{\perp}\right), \quad \Sigma_{b}\left(z_{*}, z_{\perp}\right) \equiv\left[-\infty_{*}, z_{*}\right]_{z} \psi_{b}\left(z_{*}, z_{\perp}\right)
$$

and $U_{\mu v}\left(V_{\mu \nu}\right)$ is a shorthand notation of the strength tensor of fields $U(V)$.

The perturbative solution has the same form (3.5) and (3.6), but zero-order approximation for fields is now defined as

$$
\begin{aligned}
& g \mathscr{A}_{\mu}^{[0]}(x)=U_{\mu}\left(x_{\bullet}, x_{\perp}\right)+V_{\mu}\left(x_{*}, x_{\perp}\right) \\
& \Psi^{[0]}(x)=\Sigma_{a}\left(x_{\bullet}, x_{\perp}\right)+\Sigma_{b}\left(x_{*}, x_{\perp}\right)
\end{aligned}
$$

The corresponding expressions for strength tensors in the zero-order approximation are

$$
\begin{array}{rlrl}
g \mathscr{F}_{\bullet i}^{[0]}=U_{\bullet i}+V_{\bullet i}-i\left[U_{\bullet}, V_{i}\right], & g \mathscr{F}_{* i}^{[0]} & =U_{* i}+V_{* i}-i\left[V_{*}, U_{i}\right] \\
g \mathscr{F}_{* \bullet}^{[0]}=U_{* \bullet}+V_{* \bullet}+i\left[U_{\bullet}, V_{*}\right], & g \mathscr{F}_{i j}^{[0]}=U_{i j}+V_{i j}-i\left[U_{i}, V_{j}\right]+i\left[U_{j}, V_{i}\right]
\end{array}
$$


Using parametrization of the background fields (4.4) it is possible to separate operators in perturbative solution of the equations of motion (3.5) and (3.6) based on power counting of parameter $m_{\perp}^{2} / s$. Let us start with the linear term. In terms of background fields it has the following form:

$$
\begin{aligned}
& L_{i}^{a} \equiv \mathscr{D}^{\mu} \mathscr{F}_{\mu i}^{[0] a}+g \bar{\Psi}^{[0]} \gamma_{i} t^{a} \Psi^{[0]}=L_{i}^{(0) a}+L_{i}^{(1) a} \\
& L_{i}^{(0) a}=-\frac{i}{g}\left[U^{j a b} V_{j i}^{b}+V^{j a b} U_{j i}^{b}+\mathscr{D}_{j}^{a b}\left(U^{j b c} V_{i}^{c}+V^{j b c} U_{i}^{c}\right)\right] \\
& -\frac{2 i}{g s}\left(U_{* \bullet}^{a b} V_{i}^{b}-V_{* \bullet}^{a b} U_{i}^{b}\right)+g \bar{\Sigma}_{a} t^{a} \gamma_{i} \Sigma_{b}+g \bar{\Sigma}_{b} t^{a} \gamma_{i} \Sigma_{a} \\
& L_{i}^{(1) a}=-\frac{2 i}{g s}\left[U_{\bullet}^{a b} V_{* i}^{b}+V_{*}^{a b} U_{\bullet i}^{b}-i\left\{U_{\bullet}, V_{*}\right\}^{a b} U_{i}^{b}-i\left\{V_{*}, U_{\bullet}\right\}^{a b} V_{i}^{b}\right] \\
& L_{\bullet}^{a} \equiv \mathscr{D}^{\mu} \mathscr{F}_{\mu \bullet}^{[0] a}+g \bar{\Psi}^{[0]} \gamma_{\bullet} t^{a} \Psi^{[0]}=L_{\bullet}^{(-1) a}+L_{\bullet}^{(0) a}+L_{\bullet}^{(1) a}, \quad L_{\bullet}^{(-1) a}=\frac{i}{g} U^{j a b} V_{\bullet j}^{b} \\
& L_{\bullet}^{(0) a}=\frac{i}{g} V^{j a b} U_{\bullet j}^{b}+\frac{i}{g} \mathscr{D}^{j a b} U_{\bullet}^{b c} V_{j}^{c}+g \bar{\Sigma}_{a} t^{a} \gamma_{\bullet} \Sigma_{b}+g \bar{\Sigma}_{b} t^{a} \gamma_{\bullet} \Sigma_{a}-\frac{4 i}{g s} U_{\bullet}^{a b} V_{* \bullet}^{b} \\
& L_{\bullet}^{(1) a}=\frac{2}{g s}\left(U_{\bullet} U_{\bullet}\right)^{a b} V_{*}^{b} \\
& L_{*}^{a} \equiv \mathscr{D}^{\mu} \mathscr{F}_{\mu *}^{[0] a}+g \bar{\Psi}^{[0]} \gamma_{*} t^{a} \Psi^{[0]}=L_{*}^{(-1) a}+L_{*}^{(0) a}+L_{*}^{(1) a}, \quad L_{*}^{(-1) a}=\frac{i}{g} V^{j a b} U_{* j}^{b} \\
& L_{*}^{(0) a}=\frac{i}{g} U^{j a b} V_{* j}^{b}+\frac{i}{g} \mathscr{D}^{j a b} V_{*}^{b c} U_{j}^{c}+g \bar{\Sigma}_{a} t^{a} \gamma_{*} \Sigma_{b}+g \bar{\Sigma}_{b} t^{a} \gamma_{*} \Sigma_{a}+\frac{4 i}{g s} V_{*}^{a b} U_{* \bullet}^{b} \\
& L_{*}^{(1) a}=\frac{2}{g s}\left(V_{*} V_{*}\right)^{a b} U_{\bullet}^{b} \\
& L_{\psi} \equiv \not P \Psi^{[0]}=L_{\psi}^{(0)}+L_{\psi}^{(1)} \\
& L_{\psi}^{(0)}=\gamma^{i} U_{i} \Sigma_{b}+\gamma^{i} V_{i} \Sigma_{a}, \quad L_{\psi}^{(1)}=\frac{2}{s} \not p_{2} U_{\bullet} \Sigma_{b}+\frac{2}{s} \not p_{1} V_{*} \Sigma_{a}
\end{aligned}
$$

Using parametrization of the background fields (4.4) it is straightforward to obtain the following power counting for the linear term:

$$
\begin{aligned}
& L_{i}^{(0)} \sim m_{\perp}^{3}, \quad L_{i}^{(1)} \sim \frac{m_{\perp}^{5}}{s} \\
& L_{\bullet}^{(-1)} \sim L_{*}^{(-1)} \sim s m_{\perp}^{2}, \quad L_{\bullet}^{(0)} \sim L_{*}^{(0)} \sim m_{\perp}^{4}, \quad L_{\bullet}^{(1)} \sim L_{*}^{(1)} \sim \frac{m_{\perp}^{6}}{s} \\
& L_{\psi}^{(0)} \sim m_{\perp}^{5 / 2}, \quad L_{\psi}^{(1)} \sim \frac{m^{9 / 2}}{s}
\end{aligned}
$$

Using this result and expansion of the propagator in the background field (3.9) one can obtain the following decomposition of the perturbative solution (3.5) and power counting for different components of the fields:

$$
\begin{aligned}
& A_{\bullet}^{[0]}+A_{\bullet}^{[1]}=A_{\bullet}^{(0)}+A_{\bullet}^{(1)}+O\left(\frac{m_{\perp}^{6}}{s^{2}}\right) \\
& A_{\bullet}^{(0) a}=A_{\bullet}^{([1] 0) a}+\frac{1}{g} U_{\bullet}^{a}=\frac{1}{p_{\|}^{2}} L_{\bullet}^{(-1) a}+\frac{1}{g} U_{\bullet}^{a}=\frac{1}{g} U_{\bullet}^{a}+\frac{1}{2 g \alpha} U_{j}^{a b} V^{j b} \sim m_{\perp}^{2} \\
& A_{\bullet}^{(1) a}=\frac{1}{p_{\|}^{2}} L_{\bullet}^{(0) a}+\frac{1}{2 g p_{\|}^{2}}\left(\left(\left\{\alpha, U_{\bullet}\right\}+\left\{\beta, V_{*}\right\}-\mathscr{P}_{\perp}^{2}\right) V^{j}\right)^{a b} \frac{1}{\alpha} U_{j}^{b}-2 i \frac{1}{p_{\|}^{2}}\left(V_{\bullet}^{i}\right)^{a b} A_{i}^{(1) b} \\
& +\frac{4 i}{s} \frac{1}{p_{\|}^{2}}\left(U_{* \bullet}+V_{* \bullet}\right)^{a b} \frac{1}{p_{\|}^{2}} L_{\bullet}^{(-1) b}-\frac{i g f^{a b c}}{\alpha s} A_{*}^{([1] 0) b} A_{\bullet}^{([1] 0) c}-\frac{1}{p_{\|}^{2}} A_{\bullet}^{([1] 0) a b} U_{j}^{b c} V^{c j} \sim \frac{m_{\perp}^{4}}{s}
\end{aligned}
$$


where

$$
\begin{aligned}
& A_{\bullet}^{([1] 0) a} \equiv \frac{1}{p_{\|}^{2}} L_{\bullet}^{(-1) a}=\frac{i}{2 \alpha g} f^{a b c} U_{j}^{b} V^{c j}, \quad A_{*}^{([1] 0) a} \equiv \frac{1}{p_{\|}^{2}} L_{*}^{(-1) a}=-\frac{i}{2 \beta g} f^{a b c} U_{j}^{b} V^{c j} \\
& \Rightarrow \mathscr{D}_{*} A_{\bullet}^{([1] 0) a}-\mathscr{D}_{\bullet} A_{*}^{([1] 0) a}=\frac{s}{2 g} f^{a b c} U_{j}^{b} V^{c j}+O\left(m_{\perp}^{2}\right) \\
& A_{*}^{[0]}+A_{*}^{[1]}=A_{*}^{(0)}+A_{*}^{(1)}+O\left(\frac{m_{\perp}^{6}}{s^{2}}\right) \\
& A_{*}^{(0) a}=A_{*}^{([1] 0) a}+\frac{1}{g} V_{*}^{a}=\frac{1}{p_{\|}^{2}} L_{*}^{(-1) a}+\frac{1}{g} V_{*}^{a}=\frac{1}{g} V_{*}^{a}-\frac{1}{2 g \beta} U_{j}^{a b} V^{j b} \sim m_{\perp}^{2} \\
& A_{*}^{(1) a}=\frac{1}{p_{\|}^{2}} L_{*}^{(0) a}+\frac{1}{2 g p_{\|}^{2}}\left(\left(\left\{\alpha, U_{\bullet}\right\}+\left\{\beta, V_{*}\right\}-\mathscr{P}_{\perp}^{2}\right) U^{j}\right)^{a b} \frac{1}{\beta} V_{j}^{b}-2 i \frac{1}{p_{\|}^{2}}\left(U_{*}^{i}\right)^{a b} A_{i}^{(1) b} \\
& -\frac{4 i}{s} \frac{1}{p_{\|}^{2}}\left(U_{* \bullet}+V_{* \bullet}\right)^{a b} A_{*}^{([1] 0) b}+\frac{i g f^{a b c}}{\beta s} A_{*}^{([1] 0) b} A_{\bullet}^{([1] 0) c}-\frac{1}{p_{\|}^{2}} A_{*}^{([1] 0) a b} V_{j}^{b c} U^{c j} \sim \frac{m_{\perp}^{4}}{s} \\
& A_{i}^{[0]}=A_{i}^{(0)}=\frac{1}{g}\left(U_{i}+V_{i}\right) \sim m_{\perp} \\
& A_{i}^{[1]}+A_{i}^{[2]}=A_{i}^{(1)}+A_{i}^{(2)}+O\left(\frac{m_{\perp}^{7}}{s^{3}}\right), \quad A_{i}^{(1)}=\frac{1}{p_{\|}^{2}} \tilde{L}_{i}^{(0)} \sim \frac{m_{\perp}^{3}}{s} \\
& A_{i}^{(2) a}=\frac{1}{p_{\|}^{2}} \tilde{L}_{i}^{(1) a}+\frac{1}{p_{\|}^{2}}\left(\mathscr{P} \mathscr{P}_{\perp}^{2}-\left\{\alpha, U_{\bullet}\right\}-\left\{\beta, V_{*}\right\}\right)^{a b} A_{i}^{(1) b}-2 i \frac{1}{p_{\|}^{2}}\left(\mathscr{F}_{i}^{[0] k}\right)^{a b} A_{k}^{(1) b}+\ldots \sim \frac{m_{\perp}^{5}}{s^{2}}
\end{aligned}
$$

where

$$
\tilde{L}_{i}^{(0)}=L_{i}^{(0)}+\frac{4 i}{s}\left(V_{\bullet i} \frac{1}{p_{\|}^{2}} L_{*}^{(-1)}+U_{* i} \frac{1}{p_{\|}^{2}} L_{\bullet}^{(-1)}\right)=L_{i}^{(0)}-\frac{2 i}{g s}\left(V_{\bullet} U^{j}\right)^{a b} \frac{1}{\beta} V_{j}^{b}-\frac{2 i}{g s}\left(U_{* i} V^{j}\right)^{a b} \frac{1}{\alpha} U_{j}^{b}
$$

From this result it is straightforward to derive power counting for strength tensor. Here we present first few leading terms which we need for calculation of the hadronic tensor (1.3):

$$
\begin{aligned}
& g F_{\bullet i}^{(-1) a}(x)=V_{\bullet i}^{a}(x), \quad g F_{* i}^{(-1) a}(x)=U_{* i}^{a}(x) \\
& g F_{\bullet i}^{(0) a}(x)=U_{\bullet i}^{a}(x)-i U_{\bullet}^{a b}(x) V_{i}^{b}(x)-\frac{i g}{2 \alpha} \tilde{L}_{i}^{(0) a}(x)+\mathscr{D}_{i}^{a b} V_{j}^{b c}(x) \frac{1}{2 \alpha} U^{c j}(x) \\
& g F_{* i}^{(0) a}(x)=V_{* i}^{a}(x)-i V_{*}^{a b}(x) U_{i}^{b}(x)-\frac{i g}{2 \beta} \tilde{L}_{i}^{(0) a}(x)+\mathscr{D}_{i}^{a b} U_{j}^{b c}(x) \frac{1}{2 \beta} V^{c j}(x) \\
& g F_{* \bullet}^{(-1) a}(x)=U_{* \bullet}^{a}(x)+V_{* \bullet}^{a}(x)-\frac{i s}{2} U_{j}^{a b}(x) V^{b j}(x) \\
& g F_{i k}^{(0) a}(x)=U_{i k}^{a}(x)+V_{i k}^{a}(x)-i\left(U_{i}^{a b}(x) V_{k}^{b}(x)-i \leftrightarrow k\right)
\end{aligned}
$$

Next we substitute this operators into the product

$$
F^{2}(x) \equiv F_{\mu v}^{a}(x) F^{a \mu v}(x)=\frac{8}{s} F_{\bullet i}^{a}(x) F_{*}^{a i}(x)+F_{i k}^{a}(x) F^{a i k}(x)-\frac{8}{s^{2}} F_{* \bullet}^{a}(x) F_{* \bullet}^{a}(x)
$$


which yields the following form of two leading terms

$$
\begin{aligned}
\left(F^{2}(x)\right)^{(-1)}= & \frac{8}{s g^{2}} U_{* i}^{a} V_{\bullet}^{a i} \\
\left(F^{2}(x)\right)^{(0)}= & F_{i k}^{(0) a}(x) F^{(0) a i k}-\frac{8}{s^{2}} F_{* \bullet}^{(-1) a}(x) F_{* \bullet}^{(-1) a}(x) \\
& +\frac{8}{s g} V_{\bullet}^{a i}(x) F_{* i}^{(0) a}(x)+\frac{8}{s g} U_{*}^{a i}(x) F_{\bullet i}^{(0) a}(x)
\end{aligned}
$$

It is easy to see that contribution to hadronic tensor proportional to $\left(F^{2}(x)\right)^{(-1)}\left(F^{2}(0)\right)^{(-1)}$ is constructed from a product of two TMD operator and represents a standard TMD factorization result.

To calculate power correction to the leading TMD operator we should consider

$$
\begin{aligned}
& \left(F^{2}(x)\right)^{(0)}\left(F^{2}(0)\right)^{(-1)}+(x \leftrightarrow 0)=\left[F_{i k}^{(0) a}(x) F^{(0) a i k}(x)-\frac{8}{s^{2}} F_{* \bullet}^{(-1) a}(x) F_{* \bullet}^{(-1) a}(x)\right. \\
& \left.+\frac{8}{s g} V_{\bullet}^{a i}(x) F_{* i}^{(0) a}(x)+\frac{8}{s g} U_{*}^{a i}(x) F_{\bullet i}^{(0) a}(x)\right] \frac{8}{s g^{2}} U_{* i}^{a}(0) V_{\bullet}^{a i}(0)+(x \leftrightarrow 0)
\end{aligned}
$$

There are several terms in this contribution. However, it is possible to show, see [12], that most of them are suppressed as $1 / s$ after Fourier transformation in hadronic tensor (1.3). The only term which is suppressed as $1 / Q^{2}$ comes from $U_{j}^{a b}(x) V^{b j}(x)$ operator in $F_{* \bullet}^{(-1) a}(x)$. This term provides a leading power correction to gluon TMD factorization at $s \gg Q^{2} \gg Q_{\perp}^{2} \gg m_{\perp}^{2}$.

If we keep only this term in eq. (4.18) we get

$$
g^{2} F^{2}(x)=\frac{8}{s} U_{*}^{a i}(x) V_{\bullet i}^{a}(x)+2 f^{m a c} f^{m b d} \Delta^{i j, k l} U_{i}^{a}(x) U_{j}^{b}(x) V_{k}^{c}(x) V_{l}^{d}(x)+\ldots
$$

where

$$
\Delta^{i j, k l} \equiv g^{i j} g^{k l}-g^{i k} g^{j l}-g^{i l} g^{j k}
$$

As a result the hadronic tensor is proportional to

$$
\begin{aligned}
g^{4} F^{2}(x) F^{2}(0) & =\frac{64}{s^{2}} U_{*}^{m i}(x) V_{\bullet i}^{m}(x) U_{*}^{n j}(0) V_{\bullet j}^{n}(0) \\
+ & \frac{16}{s} f^{m a c} f^{m b d} \Delta^{i j, k l}\left[U_{i}^{a}(x) U_{j}^{b}(x) V_{k}^{c}(x) V_{l}^{d}(x) U_{*}^{n r}(0) V_{\bullet r}^{n}(0)\right. \\
& \left.\quad+U_{*}^{n r}(x) V_{\bullet r}^{n}(x) U_{i}^{a}(0) U_{j}^{b}(0) V_{k}^{c}(0) V_{l}^{d}(0)\right]
\end{aligned}
$$

Finally, to calculate hadronic tensor we substitute approximation (4.23) to Eq. (1.3). Fourier transformation and separation of color singlets yields

$$
\begin{aligned}
& W\left(p_{A}, p_{B}, q\right)=\frac{64 / s^{2}}{N_{c}^{2}-1} \int d^{2} x_{\perp} \frac{2}{s} \int d x_{\bullet} d x_{*} \cos \left(\alpha_{q} x_{\bullet}+\beta_{q} x_{*}-(q, x)_{\perp}\right) \\
& \times\left\{\left\langle p_{A}\left|\hat{U}_{*}^{m i}\left(x_{\bullet}, x_{\perp}\right) \hat{U}_{*}^{m j}(0)\right| p_{A}\right\rangle\left\langle p_{B}\left|\hat{V}_{\bullet i}^{n}\left(x_{*}, x_{\perp}\right) \hat{V}_{\bullet j}^{n}(0)\right| p_{B}\right\rangle\right. \\
&-\frac{4 N_{c}^{2}}{N_{c}^{2}-4} \frac{\Delta^{i j, k l}}{Q^{2}} \frac{2}{s} \int_{-\infty}^{x_{\bullet}} d x_{\bullet}^{\prime} d^{a b c}\left\langle p_{A}\left|\hat{U}_{* i}^{a}\left(x_{\bullet}, x_{\perp}\right) \hat{U}_{* j}^{b}\left(x_{\bullet}^{\prime}, x_{\perp}\right) \hat{U}_{* r}^{c}(0)\right| p_{A}\right\rangle \\
&\left.\times \frac{2}{s} \int_{-\infty}^{x_{*}} d x_{*}^{\prime} d^{m p q}\left\langle p_{B}\left|\hat{V}_{\bullet k}^{m}\left(x_{*}, x_{\perp}\right) \hat{V}_{\bullet l}^{p}\left(x_{*}^{\prime}, x_{\perp}\right) \hat{V}_{\bullet}^{q r}(0)\right| p_{B}\right\rangle\right\}
\end{aligned}
$$




\section{Conclusions}

Using rapidity factorization approach we calculated hadronic tensor for scalar particle production (e.g. Higgs) by gluon-gluon fusion in the hadron-hadron scattering. Eq. (4.24) is the main result of this paper. It has two terms both of which have factorized form. The first term is a leading TMD result, while the second one is the leading higher-twist correction.

The author is grateful to J.C. Collins, S. Dawson, A. Kovner, D. Neill, A. Prokudin, T. Rogers, and R. Venugopalan for valuable discussions. This material is based upon work supported by the U.S. Department of Energy, Office of Science, Office of Nuclear Physics under contracts DEAC02-98CH10886 and DE-AC05-06OR23177.

\section{References}

[1] J. C. Collins, D. E. Soper Nucl. Phys. B194, 445 (1982);

[2] X. Ji, Jian-Ping Ma, and F. Yuan, Phys. Rev. D71, 034005 (2005);

[3] J. C. Collins, Foundations of Perturbative QCD (Cambridge University Press, Cambridge, 2011);

[4] M.G. Echevarria, A. Idilbi, and I. Scimeni, JHEP 07, 002 (2012);

[5] I. Balitsky, Nucl. Phys. B463, 99 (1996); Phys. Rev. D60, 014020 (1999);

[6] I. Balitsky, Phys. Rev. D72, 074027 (2005);

[7] Y. Hatta, E. Iancu, L. McLerran, A. Stasto and D. N. Triantafyllopoulos, Nucl. Phys. A764, 423 (2006);

[8] Y. Hatta, E. Iancu, L. McLerran and A. Stasto, Nucl. Phys. A762, 272 (2005);

[9] T. Altinoluk, A. Kovner, M. Lublinsky and J. Peressutti, JHEP 03, 109 (2009);

[10] I. I. Balitsky and V. M. Braun, Phys. Lett. B222, 123 (1989);

[11] I. I. Balitsky and V. M. Braun, Nucl. Phys. B361, 93 (1991);

[12] I. Balitsky and A. Tarasov, JHEP 07095 (2017). 